\title{
Research on the impact of crowd queuing behavior on construction personnel evacuation
}

\author{
Zeng Meiting ${ }^{1}$, Zhao Daoliang ${ }^{2 *}$, Yang $\mathrm{Li}^{3}$, Lu Yun ${ }^{4}$, Shi Lei ${ }^{5}$, and $\mathrm{ZhaoHu}^{6}$ \\ ${ }^{123456}$ College of urban construction and safety engineering, Shanghai institute of technology, Shanghai, 201418, China
}

\begin{abstract}
The evacuation channel is an important way to connect the room and the security exit. This paper studies the impact of queuing behavior on the evacuation of people in the building channel. In this paper, Considering the factors of evacuation channel width, personnel density and speed, queuing form, different queuing queues, etc., using Massmotion software simulation. The research shows that for different queuing methods, the evacuation efficiency of queuing by shares is less affected by the width of the channel, there is a critical channel width for the evenly divided queues, and the evacuation efficiency of the different queuing queues is inconsistent; under different widths, the personnel density and speed have different evacuation efficiency Law of influence. The research in this paper can provide a certain reference for the design of building evacuation.
\end{abstract}

\section{Foreword}

As the population density of cities continues to increase, new buildings with the main goal of accommodating more people are the current focus of many cities. Many scholars [1-6] conducted in-depth research on the evacuation model of building entrances and exits. $\mathrm{Lu}$ Hui[7] and others conducted research on the impact of building exit obstacles on evacuation. Liu Lei[3] and others believed that In the exit building, people will choose the exit by the evacuation distance and the exit queue time. Liu Huiying [8] et al. concluded that in the case of multiple exits in the building, the larger the exit width, the more people choose the exit. Chu Guanquan [9] et al. think the exit width The size of the exit is the main influencing factor for the congestion of the exit, and the influence of exit width on evacuation is limited by the evacuation preparation time.

At present, there are few researches on the queuing behavior of people in building passages. This paper uses software simulation, considering different queuing methods and physical factors of the passage, and derives the influence law of queuing people in the passage on evacuation.

\section{Evacuation model}

\subsection{Model parameter setting}

The "Unified Standards for Civil Building Design" sets the width of the flow of people per share to $0.55 \mathrm{~m}$, and puts forward an increase of $(0 \sim 0.15) \mathrm{m}$ of the human body swing requirement for the flow of people per share, and the minimum width of the fire lane is not less than $4 \mathrm{~m}$, and queues for investigators For the law of influence on evacuation, the width of the evacuation channel in this article is set to $0.7 \mathrm{~m}-4 \mathrm{~m}$, and the "Code for Fire Protection Design of Buildings" stipulates that the linear distance from any point in a public building to the nearest evacuation door or safety exit should not be greater than $30 \mathrm{~m}$. It should be greater than $40 \mathrm{~m}$. In order to facilitate the observation of the evacuation dynamics, the evacuation channel length is set to $40 \mathrm{~m}$. Figure 1 shows the software evacuation model of 4 line.

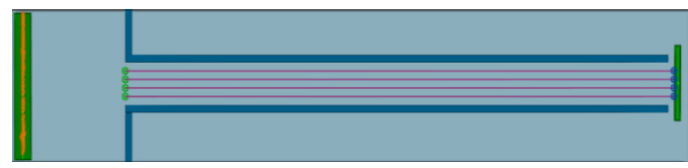

Fig. 1 Software evacuation model

\subsection{The crowd set}

This paper selects the default personnel characteristic parameters of the software, sets the maximum moving speed of the individual to $2 \mathrm{~m} / \mathrm{s}$, the minimum moving speed to $0.6 \mathrm{~m} / \mathrm{s}$, the shoulder width of the personnel is set to $0.25 \mathrm{~m}$, and the normal distribution selects the Fruin round trip character attribute, which can be based on density. Constrain the speed of the individual. In order to reduce the experimental error caused by the random allocation of the software during the experiment, the data this time is taken from the average of three simulations. 


\section{Queuing Mode}

\subsection{The setting}

There are two queuing methods for queuing in the aisle, as shown in Figure 2. Taking three queuing lines as an example, set the channel width to $6 \mathrm{a}$, then according to the queuing line equalization method, the distance between the queuing lines is $1.5 \mathrm{a}$; according to the three flow of people, There are two diversion lines, each queuing line is set at the position of the center line of the flow of people per share, the distance between adjacent queuing lines is $2 \mathrm{a}$, and the width of the queuing lines on both sides from the channel wall is aa.
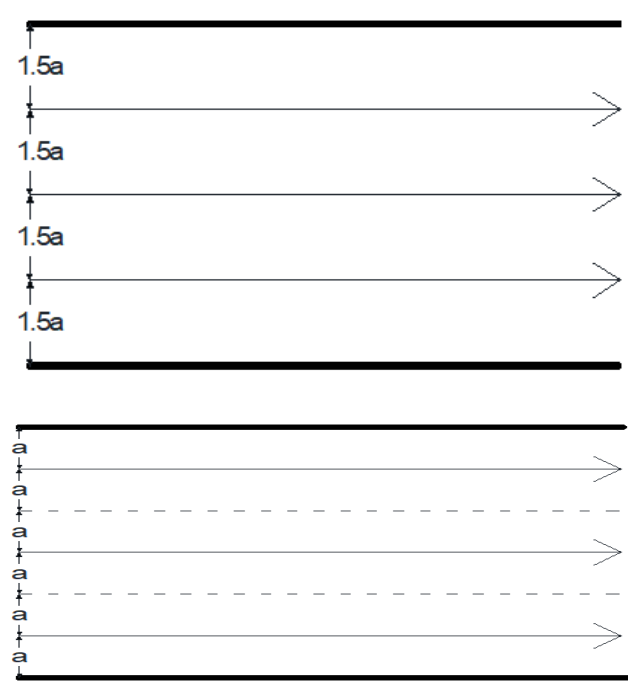

corridor

corridor

Queue line

(a) Line up evenly (b) Line up by shares

Fig. 2 Schematic diagram of different queuing modes of 3 lines

\subsection{Influence of different queuing modes on evacuation}

The pedestrian flow width per share was set as $0.6 \mathrm{~m}$, and the personnel speed followed the normal distribution (mean $1.35 \mathrm{~m} / \mathrm{s}$, standard deviation $0.25 \mathrm{~m} / \mathrm{s}$ ). By changing the channel width, the influences of the two queuing modes under the three queuing lines on evacuation were obtained.

It can be seen from Figure 2 that when there are few evacuees, no matter what queuing mode is adopted, increasing the channel width will not cause the change of evacuation time. This is because when there are few people to be evacuated, there will be no personnel congestion at the entrance of the passage and orderly evacuation can take place. Therefore, no matter how to change the width of the passage, the evacuees on each queuing line will not change much and the evacuation efficiency will not be significantly improved.
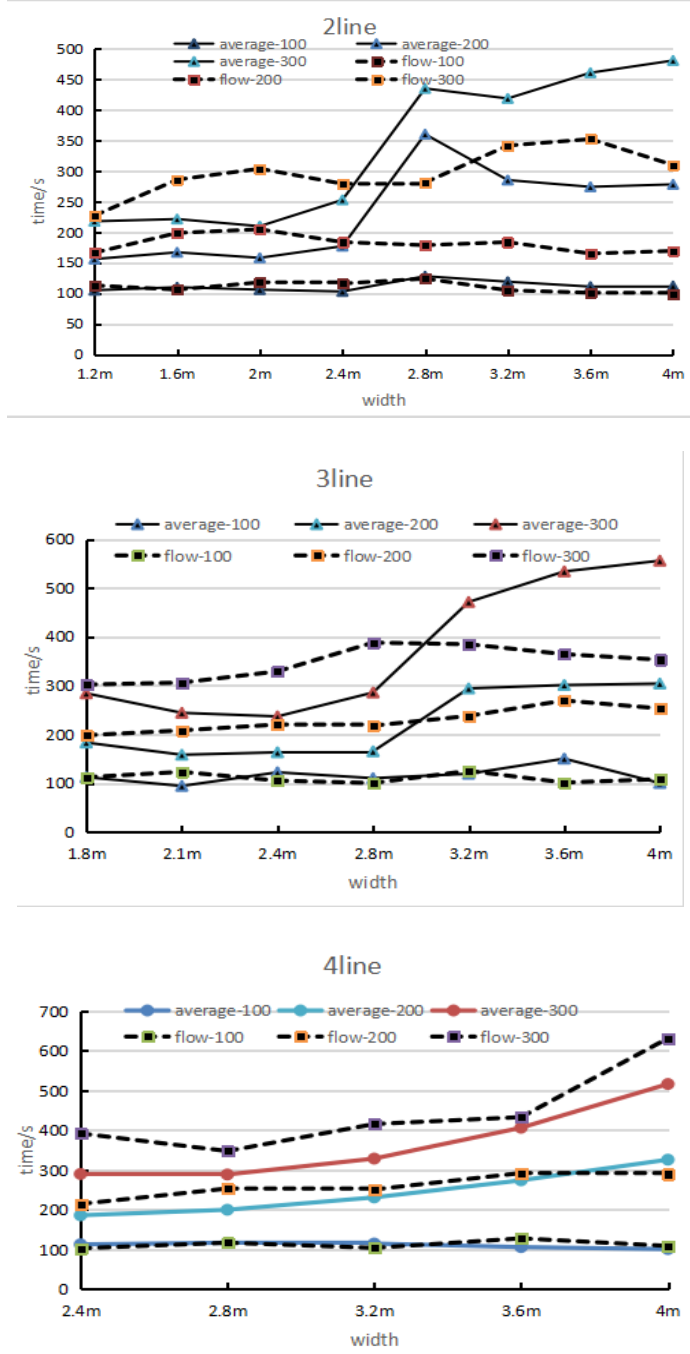

Fig. 3 Variation of Evacuation Time in Different Queue Lines and Different Queue Methods

When there is crowded evacuation Numbers increase gradually to the entrance, see from figure 3, under the same density, the split line, different queuing line width is a turning point, the line width is greater than $2.4 \mathrm{~m}, 2$ evacuation time increased by $42 \%, 3$ line width is greater than $3 \mathrm{~m}$, evacuation time increased by $65 \%$, when the line width is greater than $3.6 \mathrm{~m}, 4$ evacuation time increased by $71 \%$, and divided the line width is greater than the width of inflection point the evacuation time is greater than the press line. As can be seen from Figure 4, before the emergence of the width of the inflection point, the time spent in the queue by the stock line was higher than the bisecting method of the queue line because the two lines in the queue by the stock line were narrow from the channel wall and restricted the movement of the personnel. As the width of channel width increases to reach a turning point, queue line distance from channel width also increases, then the channel wall limit function abate, personnel for the choice of the path used time become the main influencing factors to the growth of the evacuation time, queue line and share the way because of the personnel of each queue line selection probability is consistent, so the researchers used when making routing time relative to the line is longer, more obvious overall 
evacuation time increase.
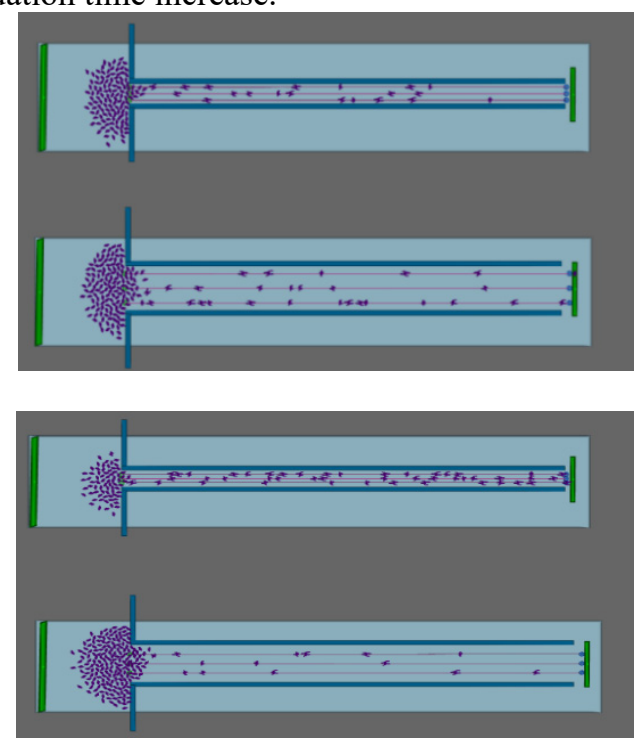

(a) Average line $\mathrm{d}=1.8 \mathrm{~m} \quad \mathrm{~d}=4.0 \mathrm{~m}$

(b) flow line $\mathrm{d}=1.8 \mathrm{~m} \mathrm{~d}=4.0 \mathrm{~m}$

Fig. 4 Dynamic Diagram of Evacuation of Three Lines with Two Queuing Methods for 300 People

In conclusion, queue evacuation has a more significant impact on evacuation efficiency in the case of crowded people. When there are fewer people to be evacuated in the evacuation channel, there is little difference in evacuation efficiency between different queuing modes. Therefore, the most convenient evacuation mode can be adopted according to the actual situation. When the number of evacuees increases and the entrance of the passage is crowded, there are inflection point widths in different queues. When the width is smaller than the width of the inflection point, evenly divided queuing should be adopted; when the width is larger than the inflection point, queuing by unit can significantly improve the evacuation efficiency.

\section{Influence of channel width on ecacuation}

Section 2.2 simulation from the queue to evacuate, channel width change will significantly affect the evacuation efficiency, for casual evacuation can be seen from the figure 5 , when the channel width is less than 2 $\mathrm{m}$, width increase can significantly reduce the evacuation time, improve the efficiency of the evacuation and evacuation, the greater the number, the more obvious the phenomenon, this is because when the channel for evacuees, easy to form the personnel crowded at the entrance of channel and queuing phenomenon formed arch structure, as shown in figure 6 , and increase the channel width, personnel in less congestion situation; When the width of the passageway is greater than $3.4 \mathrm{~m}$, the flow of people at the entrance of the passageway increases significantly, but with the increase of the walking distance, the number of people evacuated decreases significantly. The larger the width of the passageway, the shorter the high-density crowd area. Therefore, if the width of the passageway continues to increase, the improvement of crowd evacuation efficiency is not obvious.

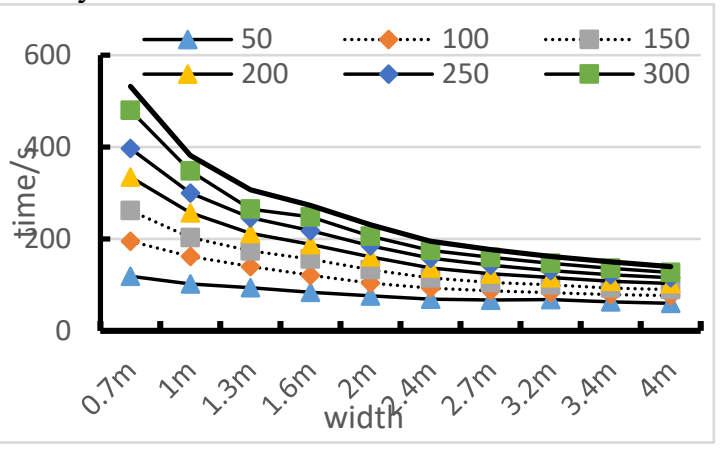

Fig. 5 Relation of random evacuation with width at different densities

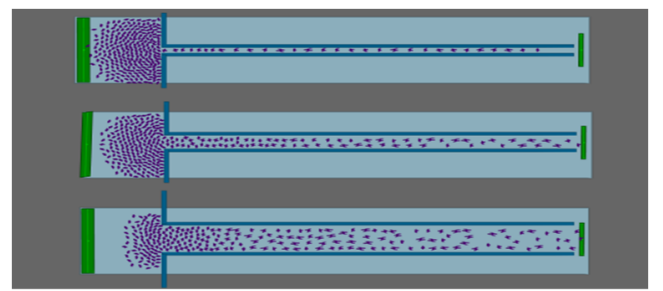

Fig. 6 Dynamic diagram of 300 people random evacuation with different widths

\section{5 conclusion}

Using Massmotion software to simulate the evacuation behavior of people in the horizontal passage of the building under the queuing situation, the following conclusions are drawn:

1) Different queuing methods will significantly affect the crowd evacuation efficiency. There is an inflection point channel width in the evacuation process. For 2line, 3line and 4line queuing, the critical evacuation width is $2.4 \mathrm{~m}, 3 \mathrm{~m}$ and $3.6 \mathrm{~m}$ respectively. When the width is larger than the inflection point, the evacuation efficiency is higher by the way of queue.

2) The number of evacuees has a linear relationship with the random evacuation time and a polynomial distribution relationship with the queuing evacuation time; Under the same passage condition, when the number of evacuees is greater than 150 , the change of evacuation time is more obvious.

\section{References}

1. Xiong Lichun, Yang Libing, Chen Jianhong, Zheng Haili, Shi Dongping (2017): Research on Optimization Model of Personnel Evacuation Based on Graph Theory and Queuing Theory [J]. Journal of Safety and Environment14(03).pp: 166-171.

2. Li Minghua, Yuan Zhenzhou, $\mathrm{Xu}$ Yan, Zang Guangzhi (2015): Research on Multi-exit Evacuation Optimization Model Based on Point Queuing Model $[\mathrm{J}]$. Transportation Systems Engineering and Information Technology15(04), pp:166-172. 
3. LIU Lei, LIU Qun, GAO Yi, et al(2011): Simulation of Evacuation Based on QuEUing Time under Multiple Exit Conditions [J]. Computer Engineering, pp:349-352.

4. Zhou D, Hu J, Gao X, et al (2019): Queueing behaviors at exit in cellular automaton model with $\mathrm{S}$ -queue[J]. International Journal of Modern Physics B, 33(8), pp:1950064.

5. Hu J, You L, Zhang H, et al (2017): Study on queueing behavior in pedestrian evacuation by extended cellular automata model[J]. Physica A Statistical Mechanics \& Its Applications.

6. Xu Guang, Qu Jun, Xia Changjun, Wang Tianhui (2010): Research on Exit Scheme of Personnel Evacuation Based on Queuing Theory [J]. Security Science and Technology (12), pp: 35-38.

7. Hui Lv, Xiangyuan Liu, Yinglong Wang, Rui Zhou(2010):Study on the Influence Law of Crowd Evacuation Exit Obstacles on Evacuation Efficiency [J]. Journal of Safety Science and Technology 16(01), pp:141-145.

8. Liu Huiying, Zhang Dezhen, Zhang Xiuguo, Ying Tang (2019): Study on Multi-exit Emergency Evacuation Model Considering Exit Width Factor [J]. pp: $1-5$. 